\section{Avaliação da atenção pré-natal em uma instituição filantrópica da cidade de São Paulo}

\section{Assessment of the process of prenatal care in a philanthropic healthcare institution in the city of São Paulo}

Márcia Duarte Koffman 1 Isabel Cristina Bonadio 2

\begin{abstract}
Objectives: to assess prenatal healthcare in a philanthropic institution in the city of São Paulo according to the Donabedian proposal.

Methods: to assess indicators pregnancy age in the beginning of prenatal care; number, procedures and consultation intervals and laboratory exams, the Kessner index as adapted by Takeda was applied. Data were obtained through auditing of 635 medical files from July 2000 to June, 2001. The significance level of statistical exams was of 5\%.

Results: prenatal medical visits median was of 6.5 $(d p=2.7)$ and pregnancy age at the beginning of prenatal care was of 20.7 weeks $(d p=7.7)$. Medical visits procedures adequacy varied from $92.6 \%$ a $97.8 \%$ and medical visits interval was $53,7 \%$ to $100 \%$. The application of the Kessner Index rated prenatal care as being adequate for $38.4 \%$ of pregnant women, inadequate for $24.7 \%$ and intermediary for $36.9 \%$. The Kessner index compounded by exams results rated prenatal care as being adequate for $35,7 \%$ of pregnant women, inadequate for $28.3 \%$ and intermediate for $35.9 \%$.

Conclusions: the type of assessment adopted is viable is allows for periodical applications aiming at adequacy improvement of the care offered.

Key words Outcome and process assessment (Health care), Prenatal care, Obstetrical nursing
\end{abstract}

1 Departamento de Enfermagem Materno-Infantil e Psiquiátrica. Escola de Enfermagem. Universidade de São Paulo. Av. Dr. Enéas de Carvalho Aguiar, 419. Cerqueira César. São Paulo, SP, Brasil. CEP:05403-000. E-mail: koiffman@ajato.com.br 2 Departamento de Enfermagem Materno-Infantil e Psiquiátrica. Escola de Enfermagem. Universidade de São Paulo. São Paulo, SP, Brasil.

\section{Resumo}

Objetivos: avaliar o processo de atendimento prénatal de uma instituição filantrópica da cidade de São Paulo, conforme a proposta de Donabedian.

Métodos: para analisar os indicadores: idade gestacional de início do pré-natal; número, procedimentos e intervalo de consultas e exames laboratoriais, aplicou-se o índice de Kessner adaptado por Takeda. Os dados foram obtidos por auditoria em 635 prontuários no período de julho de 2000 a junho de 2001. O nível de significância dos os testes estatísticos foi de $5 \%$.

Resultados: a média de consultas de pré-natal foi de $6,5(d p=2,7)$ e a da idade gestacional de início do pré-natal 20,7 semanas $(d p=7,7)$. A adequação dos procedimentos de consultas variou de $92,6 \%$ a $97,8 \%$ e o intervalo entre as consultas de 53,7\% a 100\%. A aplicação do Índice de Kessner classificou o prénatal como adequado para $38,4 \%$ das gestantes, inadequado para $24,7 \%$ e intermediário para $36,9 \%$. $O$ índice de Kessner acrescido dos resultados dos exames classificou o pré-natal em adequado para $35,7 \%$ das gestantes, inadequado para $28,3 \%$ e intermediário para $35,9 \%$.

Conclusões: o tipo de avaliação adotado é factível e permite realizações periódicas com vista à melhora da adequação dos indicadores da assistência oferecida pelo serviço.

Palavras-chave Avaliação de processo e resultado (Saúde Pública), Cuidado pré-natal, Enfermagem obstétrica 


\section{Introdução}

A assistência pré-natal de qualidade é fundamental para redução da mortalidade materna e perinatal. Garantir uma assistência adequada significa prevenir, diagnosticar e tratar os eventos indesejáveis na gestação, visando ao bem-estar da gestante e seu concepto, além de orientar para evitar problemas específicos do parto, ou mesmo, determinados cuidados imediatos ao recém-nascido.

Desse modo, consideramos importante avaliar o processo de atendimento para traçar diretrizes, a fim de otimizar os serviços e garantir uma assistência de qualidade.

No Brasil, em relação à qualidade da atenção pré-natal, observamos uma realidade cuja cobertura adequada, por si só, não garante bons resultados na redução dos coeficientes de mortalidade materna por causas obstétricas indiretas e mortalidade perinatal precoce. 1

A situação traduz a ineficácia dos serviços de saúde para garantir um atendimento adequado não apenas em relação ao número de consultas, mas também ao conteúdo do atendimento oferecido.

$\mathrm{O}$ processo de avaliação qualitativa da assistência pré-natal apresenta algumas dificuldades, pois trata da observação do resultado frente aos serviços recebidos ou que deveriam ser recebidos, acrescido o fato de que o binômio mãe-filho encontra-se inserido em um contexto socioeconômico mais amplo, capaz de influenciar o resultado desejado. 2

Outros obstáculos como a falta de informação, dificuldade para estabelecer um instrumento objetivo, simples e exeqüível para avaliação, também contribuem negativamente para esse tipo de abordagem.

Em função dessas dificuldades, em nosso meio, os estudos que visam avaliar a qualidade dos serviços são escassos, a despeito de sua importante contribuição para redução da morbimortalidade materna e perinatal. ${ }^{3}$

Assim, os trabalhos limitam-se a descrever características da atenção médica, tais como: cobertura, número de consultas pré-natais e tipo de parto. ${ }^{4}$

$\mathrm{Na}$ área da saúde, a avaliação da qualidade dos serviços envolve teorias, conceitos e práticas diferenciadas. No âmbito da assistência pré-natal, alguns trabalhos recentes, como os de Dias da Costa et al.,5 e Dias da Costa e Santos 6 têm seguido propostas de avaliação indicadas por Donabedian ${ }^{7}$ que recomenda a análise da estrutura, do processo e do resultado para estimar a qualidade dos serviços de saúde.

$\mathrm{Na}$ impossibilidade de se proceder a avaliação dos três componentes, esse autor considera o estudo do processo assistencial como a melhor forma de investigar o conteúdo da atenção oferecida no nível primário de assistência. ${ }^{7}$

A avaliação do processo analisa a qualidade da assistência em relação à disponibilidade do conhecimento técnico-científico e a relação interpessoal no momento do cuidado, representando uma medida direta.

Os resultados são avaliados pelas mudanças ocorridas no estado de saúde do indivíduo ou da população, usados na abordagens epidemiológica, na área clínica e na saúde coletiva. ${ }^{7} \mathrm{O}$ emprego de indicadores de adequação da atenção pré-natal, também, tem sido amplamente utilizado em vários estudos para investigar a qualidade desse cuidado.8-11

$\mathrm{Na}$ Região Sul brasileira, existem alguns trabalhos de avaliação da qualidade da assistência pré-natal que usam esses índices de adequação do atendimento pré-natal, sobretudo, o índice de Kessner12 e sua relação com algumas variáveis significativas à qualidade da atenção pré-natal.5,6,13

O presente estudo teve como objetivo avaliar a qualidade técnica do processo assistencial oferecido pelo serviço de pré-natal de uma instituição filantrópica da cidade de São Paulo, conveniada ao Sistema Único de Saúde (SUS), de acordo com os indicadores estabelecidos pelo Ministério da Saúde.

\section{Métodos}

O estudo foi desenvolvido no serviço de pré-natal do Amparo Maternal, onde o atendimento é de responsabilidade de enfermeiras obstétricas, docentes da Escola de Enfermagem da Universidade de São Paulo (EEUSP), São Paulo, Brasil, alunos de pósgraduação e graduação em enfermagem. As normas de funcionamento e o protocolo de tratamento são estabelecidos e assinados pelo diretor clínico da instituição. A clientela constitui-se de gestantes de baixo risco obstétrico, caracterizadas como de risco social e provenientes de diferentes regiões da cidade, de outros municípios e daquelas abrigadas no Alojamento Social da Instituição. A média diária de atendimento no pré-natal é de 20 mulheres, incluindo quatro a cinco novas gestantes por dia.

A avaliação foi conduzida por avaliadores internos, membros da equipe responsável pelo atendimento pré-natal, especificamente, uma das docentes e uma aluna de graduação da EEUSP.

A fonte de dados da pesquisa compreendeu os registros em prontuários relativos ao atendimento pré-natal de todas as gestantes matriculadas no 
serviço de julho de 2000 a junho de 2001.

A definição do período ocorreu em função da data prevista para início da coleta de dados, abril de 2002, de forma que todas as gestantes matriculadas nesse intervalo tinham concluído o acompanhamento pré-natal até o início da pesquisa. Para o período definido, selecionaram-se 635 prontuários para auditoria.

As variáveis coletadas para a caracterização sociodemográfica da população estudada foram: idade, escolaridade, ocupação da gestante e história obstétrica. Para a avaliação do processo assistencial, as variáveis incluídas foram: número de consultas realizadas no pré-natal, idade gestacional na primeira consulta, expressa em semanas; intervalo entre as consultas expresso em semanas e idade gestacional, correspondente a cada intervalo; número de vezes em que os procedimentos do exame clínico, obstétrico e o resultado de exames laboratoriais foram registrados, em separado.

Os procedimentos dos exames clínico e obstétrico analisados foram: idade gestacional, peso, pressão arterial, altura uterina, ausculta dos batimentos cardiofetais, edema e apresentação fetal. Examinamos o registro de resultado dos seguintes exames laboratoriais: hemoglobina e hematócrito $(\mathrm{Hb} / \mathrm{Ht})$, tipagem sangüínea e fator $\mathrm{Rh}(\mathrm{ABO} / \mathrm{Rh})$, sorologia para sífilis (VDRL), glicemia de jejum, exame de urina tipo I.

De acordo com o Índice de Kessner, modificado por Takeda, 14 o acompanhamento pré-natal foi considerado: adequado, quando no prontuário da gestante tivesse registro de seis ou mais consultas e o início do pré-natal antes de 20 semanas; inadequado, quando o início do pré-natal ocorreu após 28 semanas ou tivesse menos de três consultas. Em todas as demais situações, o pré-natal foi considerado intermediário.

Para obtenção de resultados mais detalhados sobre a qualidade do processo assistencial na instituição, a análise dos índices de adequação considerou os seguintes registros em prontuários: exames laboratoriais realizados pela gestante, procedimentos realizados nas consultas e o intervalo transcorrido entre uma consulta e outra, de acordo com os critérios estabelecidos a seguir.

Em relação aos exames laboratoriais, acrescentou-se ao índice de Kessner modificado o registro de alguns exames de rotina recomendados pelo MS: $\mathrm{Hb} / \mathrm{Ht}$, glicemia de jejum, VDRL, Urina I, ABO/Rh.

Para adequação da realização desses exames ao longo do acompanhamento pré-natal, a definição dos parâmetros foi um ponto que gerou dúvidas, visto que o Ministério da Saúde, em duas publicações diferentes, ambas de 2000, faz recomendações distintas em relação à solicitação dos referidos exames.

A publicação "Assistência pré-natal - Manual Técnico"15 estabelece que esses exames devem ser solicitados rotineiramente na primeira consulta prénatal sem mencionar a necessidade de sua repetição a não ser em situações específicas.

No entanto, em outra publicação, de dezembro de 2000, "Programa de Humanização do Pré-natal e Nascimento" orienta que os exames laboratoriais: $\mathrm{ABO} / \mathrm{Rh}$ e $\mathrm{Hb} / \mathrm{Ht}$ sejam solicitados na primeira consulta; VDRL, Urina I e glicemia de jejum, na primeira consulta e repetidos na trigésima semana de gestação. 16,17

Embora a segunda publicação seja mais recente, atualmente, o serviço avaliado segue as recomendações do Manual Técnico do Ministério da Saúde e não do Programa de Humanização do Pré-Natal e Nascimento. A fim de evitar resultados distorcidos, optamos por verificar a adequação do pré-natal em relação aos exames laboratoriais de rotina nas duas situações.

O Índice de Kessner associado a um registro de cada exame foi classificado como: adequado - o prénatal com seis ou mais consultas; início antes de 20 semanas de gestação e, pelo menos, um registro de todos os exames laboratoriais de rotina; inadequado - o pré-natal com menos de três consultas ou com início após 28 semanas de gestação, ou nenhum registro de exame; intermediário - todas as demais situações.

Para o Índice de Kessner associado a dois registros de exames, classificou-se como adequado o pré-natal com seis ou mais consultas, início antes de 20 semanas e, pelo menos, um registro aos exames de $\mathrm{Hb} / \mathrm{Ht}$ e $\mathrm{ABO} / \mathrm{Rh}$ e dois registros de VDRL, Urina I e glicemia de jejum; inadequado e intermediário, os mesmos critérios estabelecidos para as classificações anteriores.

Para análise dos procedimentos realizados nas consultas de pré-natal, foram acrescentados ao índice de Kessner modificado o número de vezes que os procedimentos mensuração da altura uterina(AU), definição da apresentação fetal(Apres), ausculta dos batimentos cardiofetais(BCF), avaliação de edema, idade gestacional(IG), pressão arterial(PA) e peso foram registrados. Assim, o pré-natal que apresentou seis ou mais registros dos procedimentos acima foi considerado adequado; e para três ou menos registros dos procedimentos, inadequado. Para as demais situações, intermediário.

Para a definição desses pontos de corte, consideramos que os procedimentos devem ocorrer em 
todas as consultas pré-natais, mesmo nas situações que dependa da idade gestacional, por exemplo, a ausculta dos batimentos cardiofetais e identificação da apresentação fetal, não poderiam ser identificados precocemente. Porém os registros "indefinido" e "inaudível" no prontuário mostram que tais procedimentos foram realizados ou, pelo menos, mereceram atenção durante a consulta. Mas, se na descrição da consulta não foi identificado nenhum registro a respeito do procedimento, esse foi considerado como não realizado.

Os dados relativos ao intervalo entre as consultas foram analisados separadamente, tendo como parâmetro o cronograma de atendimento para a assistência pré-natal adotado pelo serviço, conforme a publicação da Secretaria de Estado da Saúde de São Paulo, que estabelece consultas mensais até a $32^{\mathrm{a}}$ semana de gestação, quinzenais da $32^{\mathrm{a}}$ a $36^{\mathrm{a}} \mathrm{e}$ semanais, após a $36^{\mathrm{a}}$ semana. 18

Para fins deste estudo, a adequação dos intervalos entre as consultas foi, assim, definida: adequado com um espaçamento de até quatro semanas para gestantes até a $32^{\text {a }}$ semana; de até duas semanas entre 33 e 36 e até uma semana, após esta idade gestacional. Inadequado às demais situações, nas quais os intervalos excederam o espaçamento proposto.

Os dados coletados foram armazenados em banco de dados e processados nos programas Epiinfo 6.0 e SPSS.

O tratamento estatístico foi realizado pela análise descritiva das variáveis. Num segundo momento, em relação às variáveis quantitativas, a análise foi feita pela observação dos valores mínimos e máximos e cálculo de médias e desvios-padrão e medianas. Para as variáveis qualitativas (níveis dos índices calculados), as freqüências absolutas e relativas foram calculadas. Quanto à análise da hipótese de igualdade de médias entre dois grupos, o teste $t$ de Student foi usado em um nível de significância de $5 \%$.

Para identificar a associação entre as variáveis sociodemográficas e a qualidade da atenção prénatal oferecida pelo serviço, foi feita a análise estatística de variância a um fator, tendo como variável dependente a adequação do pré-natal, conforme o Índice de Kessner modificado e como variáveis independentes a idade, escolaridade e paridade das gestantes $(p<0,05)$.

Este estudo foi aprovado pelo Comitê de Ética em Pesquisa da Escola de Enfermagem da Universidade de São Paulo. O Diretor Administrativo do Amparo Maternal e as pesquisadoras assinaram um "Termo de Compromisso para Realização de Pesquisa Científica".

\section{Resultados}

No período do estudo, as gestantes que freqüentaram o serviço eram jovens, com média de 22,9 anos $(\mathrm{dp}=5,7)$, idade entre 12 e 43 anos; a escolaridade média de 7,4 anos $(\mathrm{dp}=3,0) ; 2,3 \%$ nunca tinham estudado e 1,2\% tinham mais de 12 anos de estudo.

Em relação à ocupação, 60,1\% não exerciam atividade remunerada; $12,3 \%$, eram estudantes; $43,1 \%$, donas de casa, $4,7 \%$ sem ocupação ou desempregadas $(\mathrm{N}=471$, excluídas as 110 gestantes abrigadas na instituição e 54 sem registro de ocupação no prontuário). As ocupações predominantes foram as manuais não especializadas $(24,4 \%$, $\mathrm{N}=471$ ), segundo a Escala de Prestígio Ocupacional de Aparecida Jolly Gouveia, 19 dessas, 59,1\% eram empregadas domésticas.

Figura 1

Adequação do Pré-natal segundo o registro dos procedimentos de consulta.

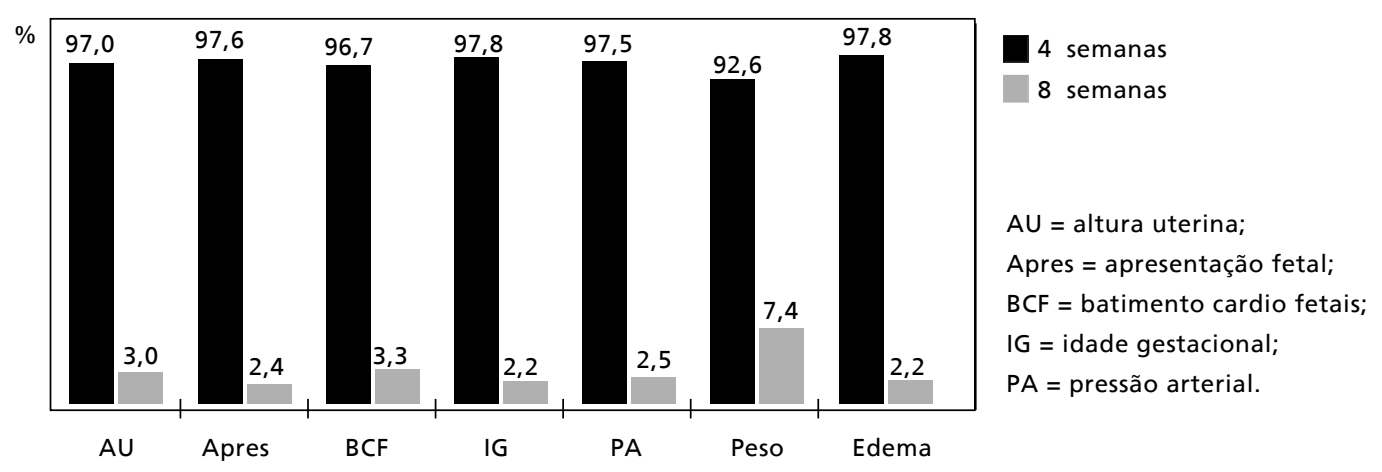


Freqüência de registros de exames laboratoriais de rotina do pré-natal. São Paulo, 2000 a 2001.

\begin{tabular}{|c|c|c|c|c|c|c|c|c|c|c|}
\hline \multirow[t]{3}{*}{ Número de registros } & \multicolumn{10}{|c|}{ Exames } \\
\hline & \multicolumn{2}{|c|}{$\mathrm{Hb} / \mathrm{Ht}$} & \multicolumn{2}{|c|}{$\mathrm{ABO} / \mathrm{Rh}$} & \multicolumn{2}{|c|}{ VDRL } & \multicolumn{2}{|c|}{ Glicemia } & \multicolumn{2}{|c|}{ Urina I } \\
\hline & $\mathrm{N}$ & $\%$ & $\mathrm{~N}$ & $\%$ & $\mathrm{~N}$ & $\%$ & $\mathrm{~N}$ & $\%$ & $\mathrm{~N}$ & $\%$ \\
\hline Nenhum & 72 & 11,3 & 89 & 14,0 & 77 & 12,1 & 104 & 16,4 & 96 & 15,1 \\
\hline Um & 517 & 81,4 & 543 & 85,5 & 557 & 87,7 & 498 & 78,4 & 402 & 63,3 \\
\hline Dois ou mais & 46 & 7,3 & 3 & 0,5 & 1 & 0,2 & 33 & 5,2 & 137 & 21,6 \\
\hline Total & 635 & 100,0 & 635 & 100,0 & 635 & 100,0 & 635 & 100,0 & 635 & 100,0 \\
\hline
\end{tabular}

Figura 2

Freqüência da adequação dos intervalos entre consultas em função da idade gestacional.

São Paulo, 2000 a 2001.

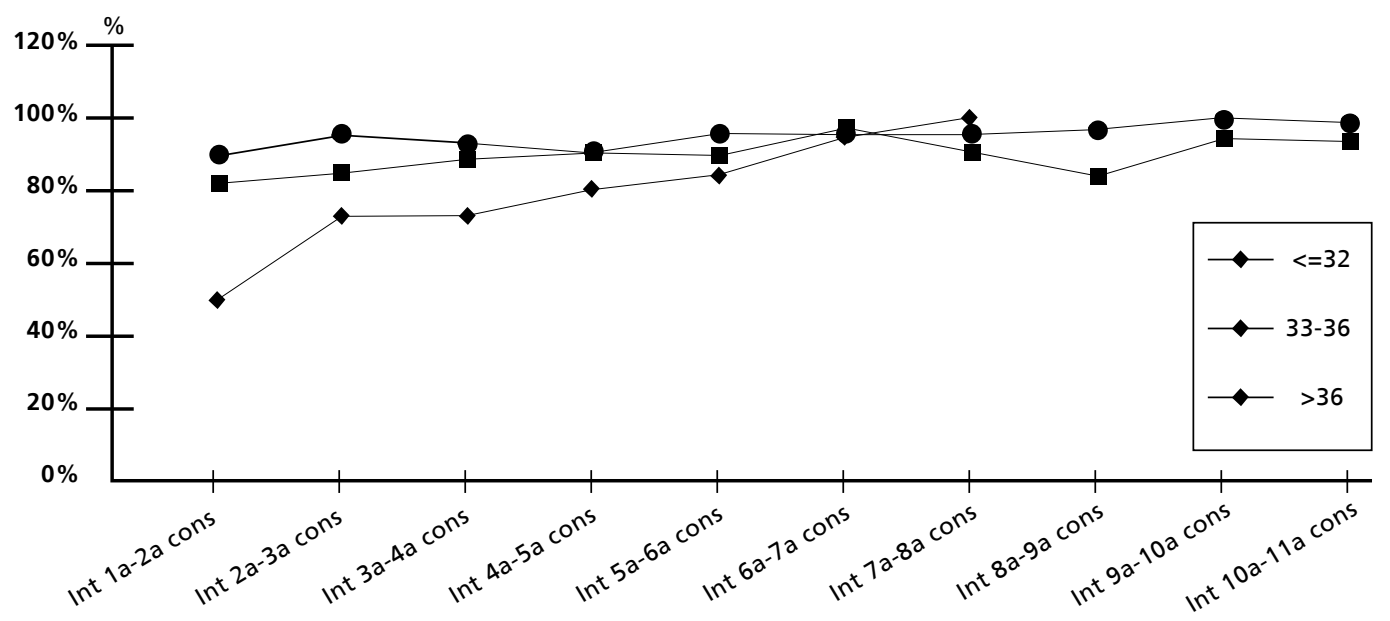

A análise da história obstétrica considerou 634 gestantes, e uma mulher não apresentava estas informações no prontuário. Em relação às gestações anteriores, $44,5 \%(\mathrm{~N}=634)$ eram primigestas; quanto à paridade, $48,9 \%(\mathrm{~N}=634)$, nulíparas. Considerando os abortamentos espontâneos e provocados, $18,1 \%$ $(\mathrm{N}=634)$ tiveram um ou mais.

A média de consultas pré-natais foi de 6,5 $(\mathrm{dp}=2,7)$, e a idade gestacional de início do atendimento de 20,7 semanas $(d p=7,7)$. Quanto ao início do acompanhamento pré-natal, 19,7\% começaram no primeiro trimestre de gestação, $57,2 \%$ no segundo e $23,1 \%$ no terceiro. Considerando o ponto de corte para a idade gestacional de início do pré-natal, proposto pelo Índice de Kessner modificado por Takeda, 14 48,1\% iniciaram o pré-natal antes de 20 semanas; $28,8 \%$ entre 20 e 27 semanas completas e $23,1 \%$ com 28 ou mais semanas de gestação.

A adequação do pré-natal, conforme o registro dos procedimentos realizados nas consultas, variou de $92,6 \%$ a $97,8 \%$. O procedimento com menor porcentual de adequação foi o peso e com maior adequação, o cálculo da idade gestacional e a verificação da presença de edema, de acordo com a Figura 1.

Conforme os registros dos exames laboratoriais 
Tabela 2

Adequação do pré-natal segundo o índice de Kessner modificado por Takeda, 14 índice de Kessner modificado e procedimentos de consulta e Índice de Kessner modificado, procedimentos de consulta e exames laboratoriais. São Paulo 2000 a 2001

\section{Número de registros}

\begin{tabular}{lcccc}
\hline Adequação & $\begin{array}{c}\text { Kessner } \\
(\mathrm{n}=635)\end{array}$ & $\begin{array}{c}\text { Kessner } \\
\text { Procedimentos } \\
(\mathrm{n}=635)\end{array}$ & $\begin{array}{c}\text { Kessner } \\
\text { Procedimentos } \\
\text { Exames 1* } \\
(\mathrm{n}=635)\end{array}$ & $\begin{array}{c}\text { Kessner } \\
\text { Procedimentos } \\
\text { Exames 2** } \\
(\mathrm{n}=635)\end{array}$ \\
\hline Adequado & 38,4 & 37,8 & $\%$ & $\%$ \\
Intermediário & 36,9 & 33,9 & 35,1 & - \\
Inadequado & 24,7 & 28,3 & 34,3 & 69,4 \\
\hline
\end{tabular}

* Exames laboratoriais de rotina segundo os indicadores estabelecidos pelo "Manual técnico" do Ministério da Saúde. 15

** Exames laboratoriais de rotina segundo os indicadores do Programa de Humanização do Pré-Natal do Ministério da Saúde.16

de rotina, a grande maioria das gestantes teve, pelo menos, um registro de cada exame ao longo do acompanhamento pré-natal, embora algumas estivessem sem nenhum exame, conforme os dados da Tabela 1.

A análise estatística das correlações entre número de registros de cada um dos exames laboratoriais, idade gestacional inicial e o número de consultas pela aplicação do coeficiente de Spearman com nível de significância de 5\%, mostrou correlação negativa e estatisticamente significativa entre idade gestacional inicial e número de registros de exames e correlação positiva e estatisticamente significativa entre número de consultas e registros de exames.

A Figura 2 mostra a adequação dos intervalos entre as consultas; os menores porcentuais ocorreram em gestantes com idade gestacional menor ou igual a 32 semanas, e o mais baixo no intervalo da primeira para a segunda consulta.

$\mathrm{O}$ teste $t$ de Student mostrou a adequação do intervalo entre as consultas, sendo menor para gestantes com idade gestacional até 32 semanas, até a terceira consulta $(p<0,01)$. As gestantes que iniciam o pré-natal, antes de 32 semanas de gestação, acabam tendo um espaçamento entre as consultas iniciais maior, quando comparadas àquelas que começam mais tardiamente.

Ao combinarmos a idade gestacional de início do pré-natal e o número de consultas (índice de Kessner modificado por Takeda), 14 o pré-natal foi classificado como adequado para $38,4 \%$ dos casos; intermediário, para $36,9 \%$ e inadequado, para $24,7 \%$.
Quando adicionamos a esses resultados os registros dos procedimentos de consulta, houve uma queda discreta no porcentual de adequação para $37,8 \%$; os pré-natais intermediários passaram para $33,9 \%$ e os inadequados subiram para $28,3 \%$.

Ao acrescentarmos a esses resultados (índice de Kessner e procedimentos de consulta), o registro de exames laboratoriais de rotina, segundo o critério do "Manual técnico" do Ministério da Saúde15 para assistência pré-natal, o porcentual de adequação caiu para $35,1 \%$, os intermediários e os inadequados subiram para $34,3 \%$ e $30,6 \%$, respectivamente. Nenhum pré-natal foi classificado como adequado, $69,4 \%$ como intermediários e $30,6 \%$ inadequados, quando esta mesma combinação foi usada aos critérios de exames laboratoriais, estabelecidos pelo Programa de Humanização do Pré-natal e Nascimento16. Esses dados estão resumidos na Tabela 2.

A análise de variância a um fator não mostrou associação estatisticamente significativa entre adequação da assistência pré-natal e idade das gestantes $(p=0,1833)$; escolaridade $(p=0,3073) \mathrm{e}$ paridade $(p=0,1146)$.

\section{Discussão}

A iniciativa de avaliar a qualidade técnica do processo assistencial da atenção pré-natal oferecida pelo Pré-Natal do Amparo Maternal passa pela necessidade de analisar e julgar as ações e atividades desenvolvidas para conhecer e modificar essa situação, mediante a tomada de novas decisões.

Nesse sentido, avaliar significa expor um valor assumido, baseado no julgamento realizado de critérios previamente definidos. Para avaliar, identificamos uma situação reconhecida como problema; e, por meio de instrumentos e referências específicas, um juízo de valor inerente a esse processo deve ser emitido.

A avaliação é uma estratégia para decidir como enfrentar e resolver problemas; serve para direcionar ou redirecionar a execução de ações e atividades e ser exercida por todos os envolvidos no planejamento e execução dessas ações. ${ }^{20}$

Em relação à resolução de problemas, a posição do avaliador externo ou interno assume papel importante no sucesso do processo avaliativo. Para a tomada de decisões, a avaliação deve se constituir em elemento efetivamente capaz de participar de processos que produzam respostas às questões apresentadas pelos que vivenciam o objeto avaliado. Essa capacidade é importante, caso seja necessária uma escolha entre um avaliador interno ou externo. 
Assim, o elemento interno passa a ter uma posição decisiva no desenrolar do processo, ainda que avaliadores externos façam parte da equipe coordenadora da avaliação. 21

A presente avaliação foi desenvolvida por agentes envolvidos no gerenciamento do serviço, avaliadores internos, possibilitando a discussão das questões apontadas no processo e a viabilização das medidas que devem ser tomadas para sua implementação.

A avaliação da adequação do acompanhamento pré-natal deu-se em três etapas. A primeira, consistiu na aplicação do Índice de Kessner adaptado por Takeda, ${ }^{14}$ bastante utilizado para mensuração da adequação da assistência pré-natal.

Conforme a autora citada, a complexidade de registro e a necessidade de um programa específico para analisar os resultados exigidos pelo índice original tornam sua aplicação impraticável em nosso meio.

As modificações preservaram a idéia principal de cruzar os dados do número de consultas com a idade gestacional de início do pré-natal, na avaliação da qualidade da assistência prevista pelo Índice de Kessner original. A esses dados, acrescentaram-se as recomendações do Ministério da Saúde e do Centro Latino-Americano de Perinatologia que definem alguns aspectos importantes à qualidade do acompanhamento pré-natal, como: idade gestacional para seu início, número de consultas e época da gestação em que essas deveriam ocorrer. 15,16

Em alguns trabalhos sobre a avaliação da atenção pré-natal, com resultados satisfatórios, 6,13 o índice modificado tem sido usado; desde 1993, pelo Serviço de Saúde Comunitária do Município de Porto Alegre, Rio Grande do Sul, para avaliar a qualidade da atenção pré-natal oferecida, demonstrando ser um bom indicador da qualidade das ações de saúde nesse contexto.

Ao avaliar o processo da atenção pré-natal do Amparo Maternal, alguns indicadores apresentaram resultados compatíveis e, em alguns aspectos, superiores aos encontrados em outros estudos sobre a assistência pré-natal no Brasil. Por outro lado, existem dificuldades estruturais relacionadas à própria instituição ou ao sistema de saúde, como um todo, que comprometem a qualidade da assistência oferecida.

No presente estudo, a média de 6,5 consultas mostrou-se superior a outros semelhantes realizados na Região Sul do País, 5,6,22 porém, inferior ao encontrado em pesquisa desenvolvida, em 1998, em Pelotas, também na Região Sul, que verificou uma média de 7,0 consultas. 13 A maioria das gestantes
$(57,2 \%)$ iniciou o pré-natal apenas no segundo trimestre de gestação, resultado similar ao dos estudos já mencionados.

A captação precoce de gestantes para início do pré-natal é de extrema importância para sua qualidade, o serviço avaliado possui algumas características específicas que, de alguma forma, podem ter influenciado esses resultados. 23,24

Em função de sua filosofia própria, o Amparo Maternal atende todas as gestantes que o procuram, independente da procedência ou idade gestacional. Recebe mulheres de todas as regiões da cidade e de outros municípios. Cerca de $75 \%$ das gestantes não reside nos bairros próximos ao serviço, ${ }^{25}$ cuja função é importante na absorção da demanda excedente de outros serviços públicos de atendimento à gestante $\mathrm{e}$ parturiente.

No presente estudo realizado com a população atendida pela Instituição, muitas gestantes procuram-no, mesmo sendo distante da região onde residem, em função da falta de vagas nas Unidades Básicas de Saúde (UBS), demora para marcação de consulta e má qualidade de atendimento. 26

Em Pelotas, Rio Grande do Sul, uma pesquisa sobre os critérios de escolha de postos de saúde, para o acompanhamento pré-natal, mostrou que $52 \%$ das gestantes não o realizaram na UBS próxima a sua residência, alegando, entre outros motivos, má qualidade do atendimento. 27

A população atendida no serviço de pré-natal do Amparo Maternal caracteriza-se pela busca tardia de assistência pré-natal, não só pela falta de conscientização sobre a importância desse atendimento, mas pelo desencanto e falta de confiança na qualidade dos serviços oferecidos pela UBS. 28

No pré-natal, os procedimentos realizados na consulta foram indicadores de melhores resultados de adequação. As ações, que fazem parte da consulta pré-natal, não demandam uma estrutura sofisticada de atendimento e dependem quase que, exclusivamente, da atuação dos recursos humanos. Isso demonstra que os profissionais envolvidos com o cuidado pré-natal valorizam a realização desses procedimentos e compreendem sua importância para a qualidade da assistência.

No Amparo Maternal, a assistência é prestada exclusivamente por enfermeiras obstétricas, alunos do curso de graduação e de pós-graduação em Enfermagem. De acordo com a Lei do Exercício Profissional da Enfermagem (Decreto n. 94.406/87), o pré-natal de baixo risco pode ser inteiramente acompanhado pela enfermeira, conforme o "Manual técnico para assistência pré-natal" do Ministério da Saúde. 15 
A Organização Mundial da Saúde, 29 na publicação "Maternidade segura - assistência ao parto normal: um guia prático", aponta a enfermeira obstétrica como o profissional mais adequado e com melhor custo de efetividade para ser responsável pela assistência à gestação e ao parto normal, incluindo, a avaliação de riscos e o reconhecimento de complicações.

Nesta avaliação, os resultados mostram não só a competência desses profissionais para o atendimento à gestação de baixo risco, mas também a necessidade de expandir sua atuação a outros serviços de atenção básica à gestante.

Na maioria das UBS, o cuidado pré-natal é feito por profissionais pouco comprometidos e numericamente insuficientes, o que resulta em um limitado oferecimento de vagas e em atendimento de baixa qualidade em relação ao conteúdo das consultas.

Desse modo, um dos fatores responsáveis pela procura do pré-natal do Amparo Maternal, com elevado porcentual de adesão das gestantes pelo serviço, independente das dificuldades enfrentadas, como acesso geográfico, é justamente o perfil de seus profissionais compromissados com a qualidade técnica, com os aspectos ético-políticos e direitos da mulher.

Quanto aos exames laboratoriais de rotina, os resultados dessa avaliação mostraram que algumas gestantes terminam o pré-natal sem nenhum registro em seus prontuários. Em grande parte, são as que iniciam o pré-natal mais tardiamente e possuem menor número de consultas. O porcentual de gestantes sem o registro do exame de sorologia para sífilis e dosagem de hemoglobina é preocupante.

Dentre as hipóteses para o baixo porcentual encontrado nas situações de gestantes com dois ou mais registros de exames laboratoriais, temos as próprias orientações do serviço.

O serviço analisado não segue as orientações do Programa de Humanização do Pré-Natal e Nascimento do Ministério da Saúde, mas, o Manual Técnico para assistência pré-natal que preconiza a realização de exames laboratoriais de rotina uma única vez ao longo do acompanhamento pré-natal, salvo em situações específicas. Seria interessante que esses critérios fossem revistos para definir qual deles realmente é o mais adequado.

Ainda em relação aos exames, é importante destacar que as dificuldades financeiras enfrentadas pela instituição afetam diretamente sua realização pelas gestantes. O laboratório que presta esses serviços de forma terceirizada não atende a grande demanda em função da limitação de quotas mensais impostas pelo SUS.
Por outro lado, a ausência de exames para rastreamento de doenças, cujo tratamento é disponível e de baixo custo, como a sífilis e anemia ferropriva, pode comprometer os resultados maternos e perinatais. Diante da situação, é necessário envidar esforços para sanar essa falha no atendimento às gestantes.

A avaliação dos intervalos entre as consultas mostrou uma preocupação maior no espaçamento do atendimento em fases mais adiantadas da gestação. As mulheres que buscam o atendimento mais precoce, têm agendamento para consulta subseqüente que, muitas vezes, ultrapassa o intervalo de 30 dias.

Seria interessante que essa situação fosse analisada com maior detalhe, para detectar as razões desses achados e sua eventual resolução. A demora no agendamento de consultas é um dos fatores de descontentamento das gestantes em relação aos serviços, além de comprometer a qualidade da assistência pré-natal.26

O critério de adequação do pré-natal pelo índice de Kessner modificado mostra uma porcentagem de pré-natais adequados $(38,4 \%)$ semelhantes a outros estudos.6,14 Destaca-se elevada porcentagem de pré-natais inadequados. $(24,7 \%)$.

No presente estudo, os resultados podem refletir tanto as limitações do serviço como as de suas usuárias. A Instituição do estudo, pela sua filosofia, não recusa vagas para qualquer mulher que o procure, independente da fase gestacional. Assim, acaba apresentando uma média de idade gestacional inicial mais elevada que outros.

Em relação às usuárias, quase sempre, realizam uma verdadeira peregrinação na busca pelo atendimento durante a gestação, até chegarem, tardiamente, ao serviço. Existem aquelas mulheres abrigadas na Instituição que a procuram quando não podem mais esconder sua condição de gestante. Esses fatores contribuem para aumento de pré-natais classificados como inadequados pelo Índice de Kessner.

Outro fator que pode ter influenciado o resultado apresentado, é a falha de registro em prontuário de algumas informações referentes ao término do prénatal pela resolução da gravidez (parto), encaminhamento de gestantes para pré-natal de alto risco ou abandono do acompanhamento.

Nesse estudo, foram excluídas as gestantes cujos prontuários apresentavam registro de encaminhamento para outro serviço, mudança de cidade e transferência de serviço para continuidade do prénatal.

Por falta de registro dessas condições no pron- 
tuário, muitas gestantes nessas situações foram incluídas no estudo. A obtenção dos dados do parto de todas as gestantes atendidas pelo ambulatório de pré-natal é difícil, pois entre $60 \%$ e $65 \%$ delas dão à luz na própria instituição.

Para fins do estudo, o pré-natal foi encerrado quando a gestante deixou de comparecer ao serviço, independente da idade gestacional em que se encontrava, salvo nas situações em que havia registro do não comparecimento por razões outras que não a resolutividade da gestação (encaminhamentos, mudança de serviço, etc.).

Os partos prematuros ( $<38$ semanas) não foram considerados como fatores de exclusão. Por essa razão, a variável relativa ao número de consultas realizadas e o índice de Kessner podem ter sido influenciados por esse viés na metodologia do estudo, pela não delimitação da idade gestacional para término do pré-natal, agravada por falta de registro em prontuário das situações com interrupção precoce da gravidez ou parto prematuro.

O uso de outros critérios de avaliação, além do número de consultas e idade gestacional de início do pré-natal, permite uma visão mais qualitativa da atenção oferecida.5,30

Ao adicionar o registro de procedimentos ao índice de Kessner modificado, conforme os critérios estabelecidos na metodologia do estudo, não observamos grande alteração na proporção entre as situações adequadas, inadequadas e intermediárias. O mesmo não ocorreu quando acrescentamos aos critérios anteriores o registro de exames laboratoriais de rotina.

\section{Referências}

1. Aerts DRG. Investigação dos óbitos perinatais e infantis: seu uso no planejamento de políticas públicas de saúde. J Pediatr [Rio J] 1997; 73: 364-6.

2. Sánchez-Centurión CAS. Avaliação da qualidade da assistência perinatal no Município de Taboão da Serra, São Paulo [dissertação mestrado] São Paulo: Faculdade de Saúde Pública da Universidade de São Paulo; 1996.

3. Adami NP, Maranhão AMSA. Qualidade dos serviços de saúde: conceitos e métodos avaliativos. Acta Paul Enferm 1995; 8: 47-55.

4. Tanaka ACD'A, Siqueira AAF, Bafile PN. Situação de saúde materna e perinatal no estado de São Paulo, Brasil. Rev Saúde Pública 1989; 23(1): 67-75.

5. Dias da Costa JS, Madeira ACC, Luz RM, Britto MAP. Auditoria médica: programa de pré-natal em posto de saúde na região Sul do Brasil. Rev Saúde Pública 2000; 34: 329-36.

6. Silveira DS, Dias da Costa JS, Santos IS. Atenção pré-natal na rede básica: uma avaliação da estrutura e do processo. Cad Saúde Pública 2001; 17: 131-9.
A avaliação realizada poderá contribuir para a melhoria da qualidade técnica do atendimento oferecido pelo serviço, a despeito da identificação dos fatores que interferem no atendimento e que se encontram fora das possibilidades de atuação de seus gestores; assim, grande parte dos problemas levantados poderá ser discutida e revista, buscando o aperfeiçoamento da assistência.

O tipo de avaliação desenvolvida nessa pesquisa, em especial, o uso de índices de adequação, constitui um método factível e eficaz. Pode ser realizado rotineiramente pelo serviço, sob as formas de amostragens periódicas, fornecendo subsídios concretos à melhora da qualidade de atendimento às gestantes.

Lembramos que, ao se iniciar um processo de avaliação de um serviço de saúde, seu objetivo é abordar os três componentes estabelecidos, segundo o referencial teórico de Donabedian: a estrutura, o processo e o resultado.7

Dessa forma, qualquer que seja o componente inicial selecionado na avaliação, é desejável analisar a inter-relação e interdependência entre esses componentes, possibilitando maior efetividade nas decisões que serão tomadas. Seria fundamental para o processo de avaliação da qualidade do serviço que fossem desenvolvidos outros estudos, analisando a estrutura e os resultados.

Os resultados encontrados fornecem dados sobre a atenção oferecida pela Instituição, além de contribuírem para divulgação de uma metodologia eficiente e exequiível de avaliação.

7. Donabedian A. The quality of care: how can it be assesed? JAMA 1988; 260: 1743-48.

8. Quick JD, Roghmann KJ. Prenatal care and pregnancy outcome in a HMO and general population: a multivariate cohort analysis. Ame J Public Health 1981; 71: 381-90.

9. Chen SP, Telleen S, Chen EH. Adequacy of prenatal care of urban high school students. Public Health Nurs 1995; 12: 47-52.

10. Kogan MD, Martin JA, Alexander GR, Kotelchuck M, Ventura SJ, Frigoletto FD. The changing pattern of prenatal care utilization in the United States, 1981-1995, using different prenatal care indices. JAMA 1998; 279: 1623-8.

11. Mikhail B. Prenatal care utilization among low-income African women. J Community Health Nurs 2000; 17: 23546.

12. Kessner DM, Singer J, Kalk CW, Schlesinger ER. Infant death: an analysis of maternal risk and health care. Washington (DC): Institute of Medicine, National Academy of Science; $1973 ;$ 50-95. 
13. Halpern R, Barros FC, Victora CG, Tomasi E. Atenção prénatal em Pelotas, Rio Grande do Sul, 1993. Cad Saúde Pública 1998; 14: 487-92.

14. Takeda S. Avaliação de unidade de atenção primária: modificação dos indicadores de saúde e qualidade da atenção [dissertação] Pelotas: Universidade Federal de Pelotas; 1993.

15. Ministério da Saúde. Secretaria de Políticas Públicas. Assistência pré-natal: manual técnico. 3. ed. Brasília (DF); 2000.

16. Ministério da Saúde. Secretaria Executiva. Programa humanização do parto. Humanização no pré-natal e nascimento. Brasília (DF); 2000.

17. Serruya SJ, Lago TDG, Cecatti JG. O panorama da atenção pré-natal no Brasil e o Programa de Humanização do Prénatal e Nascimento. Rev Saúde Matern Infant 2004; 4: 26979.

18. Monetti V, Moura JCA, Carvalho PR. Fundamentos da assistência pré-natal: para orientação dos que se dedicam à assistência médico-sanitária, à maternidade e à infância. São Paulo: Instituto de Saúde; 1971.

19.Alvarenga AT. Índice de status socioeconômico da família da mulher grávida que freqüenta o Centro de Saúde Geraldo de Paula Souza da Faculdade de Saúde Pública da Universidade de São Paulo. Rev Saúde Pública 1973; 7 : 351-67.

20. Tanaka OY, Melo C. Avaliação de programas de saúde do adolescente: um modo de fazer. São Paulo: Ed. da Universidade de São Paulo; 2001.

21. Ferreira AS. Management competencies for basic units of the Health Care System. Cienc Saúde Coletiva 2004; 9: 6976.
22. Trevisan MR, Lorenzi DRS, Araújo NM, Ésber K. Perfil da assistência pré-natal entre usuárias do Sistema Único de Saúde em Caxias do Sul. Rev Bras Ginecol Obstet 2002; 24: 293-99.

23. Barros SMO. Análise da qualidade da assistência pré-natal prestada pela enfermeira obstétrica no Amparo Maternal [dissertação mestrado]. São Paulo: Escola Paulista de Medicina; 1990.

24. Wulf KH, Steck T. The impact of timing and frequency of prenatal visits on the outcome of pregnancy in the perinatal registry of Bavária. Eur J Obstet Gynecol Reprod 1994; 57 : 79-84.

25. Tsunechiro MA, Santos EMM. Idade ao gestar como fator de risco materno e perinatal: relatório de pesquisa PIBIC/USP/CNPq]. São Paulo: Escola de Enfermagem da Universidade de São Paulo; 1997.

26. Oliveira VM. Um lugar no cuidado pré-natal: possibilidades e opções das gestantes [dissertação mestrado]. São Paulo: Escola de Enfermagem da Universidade de São Paulo; 2000.

27. Santos IS, Baroni RC, Minotto I, Klumb AG. Critérios de escolha de postos de saúde para acompanhamento pré-natal em Pelotas, RS. Rev Saúde Pública 2000; 34: 603-9.

28. Bonadio IC. Ser tratada como gente: a vivência de mulheres atendidas no serviço de pré-natal de uma instituição filantrópica. Rev Esc Enf Univ. São Paulo, 1998; 32: 9-15.

29. Organização Mundial da Saúde. Assistência ao parto normal: um guia prático. Genebra; 1996.

30. Coutinho T, Teixeira MTB, Dain S, Sayd JD, Coutinho LM. Adequação do processo de assistência pré-natal entre as usuárias do Sistema Único de Saúde em Juiz de Fora, MG. Rev Bras Gineco Obstet 2003; 25: 717-24.

Recebido em 22 de fevereiro de 2005

Versão final apresentada em 8 de agosto de 2005

Aprovado em 10 de outubro de 2005 